\title{
On the solar magnetic field polarity reversal during the activity cycle
}

\author{
E. I. Mogilevsky, K. I. Nikolskaya
}

Institute of Terrestrial Magnetism, Ionosphere and Radio Wave Propagation, RAS, Troitsk, 142190, Russia email: knikol@izmiran.troitsk.ru

Solar magnetic field polarity reversal (SMFPR) happens every 10-11 years. However untill now there is no clear understanding in what way this process is carried out. Results represented here are a by-product of the coronal hole study based on taken from Web-archive XUV corona images (Yohkoh and EIT/SOHO), white-light corona pictures(SOHO/ LASCO) and Kitt Peak coronal hole (CH) 1083 nm maps. Observations used cover the minimum, maximum and a part of declining phase of 23-d cycle (1996-2004).

Figure 1 (panel "A") displays one of numerous pairs of $\mathrm{CH}$ maps separated in time by Sun's semi-rotation (2003:June 15-28) which demonstrate two longitudinally untipodal solar hemispheres. Each hemisphere, except one of the solar poles, is fully occupied by large magnetically unipolar trans-equatorial CH complex. Boundary between two unipolar hemispheres denoted schematically on maps with dashed line points that the magnetic equator plane is strongly inclined to the solar equator. Regular growth of coronal streamer belt (CSB) inclination in the course of activity enhancement, up to $90^{\circ}$ in maximum, has been discovered in (Gulyaev \& Vanyarkha 1992) on the base of eclipse white-light corona observations. Panel "B" of Fig.1 demonstrate the streamer belt position on the SOHO/LASCO white-light corona images: for just before 23-d cycle maximum - on the right, and two years after maximum (for our case)- on the left. Besides the streamer belt position, Fig.1 reveals an observational evidence that around the activity maximum the nearly meridionally directed CSB divides the Sun into two unipolar hemispheres. Solar magnetic poles as centres of corresponding hemispheres have to be placed on the solar equator at untipodal longitudes. Magnetic pole sinchronous displacement from solar poles to the equator and farther to the opposite solar pole suggests the magnetic equator plane rotation (MEPR) around the axis lying in the solar equator plane. Signatures of such a rotation have been found in (Ikhsanov \& Ivanov 2002) as an inverse regular meridional displacement of the untipodal parts of the magnetic neutral line during the ascending phase of the cycle. Thus, represented here analysis of $\mathrm{CH}$ observations shows that during the solar cycle both magnetic poles travel from one solar pole to another and reach the opposite position in the next minimum.

As fast as activity grows, well known permanent polar coronal holes of the quiet Sun disappear and, instead, there emerge moderate - and low latitude $\mathrm{CHs}$ in form of $\mathrm{CHs}$ of the active Sun. Visibility, number, size, shape, areas, location and even temperature of such CHs vary significantly during the activity cycle (Nikolskaya 2003). Magnetic pole and polar $\mathrm{CH}$ similar behaviour in the course of the solar cicle points to their close relation. Apparently, only two $\mathrm{CHs}$ exist on the Sun, genetically connected to magnetic pole of the same polarity. When the activity evolves $\mathrm{CHs}$ migrate together with their magnetic poles and, depending on the activity phase, are observed on either solar poles or solar equator. 

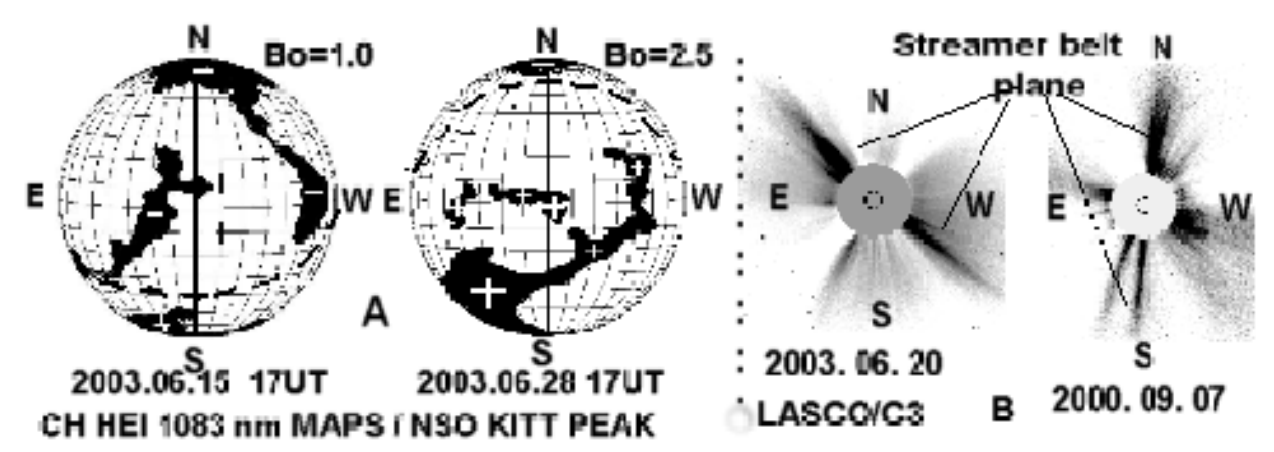

Figure 1. (A) CH maps show two untipodal unipolar solar hemispheres; dashed line designates a neutral line position; $(B)$ Streamer belt position just before (2000.09.07) and two years after (2003.06.20) activity maximum.

Results are as following.

1. Observational evidences have been presented that during SMFPR magnetic poles never vanish but travel from one solar pole to another crossing the solar equator near activity maximum.

2. SMFPR process takes the whole cycle from minimum to minimum and is completed when the north and south magnetic poles swaped positions.

3. Analysis performed here confirms the hypotesis on streamer belt plane rotation around the axis lying in the solar equator plane.

4. Only two CHs exist on the Sun coupled constantly with the same solar magmetic pole. Migrating together with magnetic poles from one solar pole to another CHs appear as PCHs of the quiet Sun or low latitude CHs of the active Sun.

5. Migration of the solar magnetic poles from one solar pole to another apparently relates to gigantic (of $\sim R_{\odot}$ in size) meridional subphotospheric circulation that is hard to understand within the macrohydrodynamics, even for relatively thin photosphere level $\left(\Delta l<0.1 R_{\odot}\right)$, because of high self-induction. Under assumption on a fractal nature of magnetized plasma large-scale subphotospheric circulation can be described as a result of the temporal variations of the distribution function of a fractal set. This causes a large value of the nonlinear magnetic super-diffusion (Mogilevsky 2001). Temporal variations of the distribution function result from a solution of the nonlinear kinetic equation with fractal derivatives for a set of fractals.

Authors are grateful to Yohkoh and SOHO/EIT/LASCO teams as well as Kitt Peak $\mathrm{CH}$ observational staff for the perfect observations we used in our study. This work is supported by RFFI GR-s 040216374 for M. and 020216199a for N.

\section{References}

Gulyaev, R. A. \& Vanyarkha, N. Ya 1992 Solar Phys., 140, 369-378.

Ikhsanov, R. N. \& Ivanov, V. G. 2002 In Intl. Conf. Proc. on Solar Activity and Cosmic Rays after a polarity Reversal of the Polar Magnetic Field of the Sun (ed. Makarov, V. I. \& V. N. Obridko), 213-220. Pulkovo, St-Peterburg.

Mogilevsky, E. I. 2001 In book Fractals on the Sun (ed. V. N. Obridko), 150p. Academic. Moscow.

Nikolskaya, K. I. 2003 In Intl. Conf. Proc. Climatic and Ecological Aspects of the Solar Activity (ed. Makarov, V. I. Yu. A. Nagovitsin and V. N. Obridko), pp. 213-220. Pulkovo, StPeterburg. 